\title{
VALIDEZ DE CONSTRUCTO DEL LSB-50 EN ADULTOS ARGENTINOS: VALIDACIÓN CRUZADA E INVARIANZA FACTORIAL
}

\author{
CONSTRUCT VALIDITY OF THE LSB-50 IN \\ ARGENTINEAN ADULTS: CROSS-VALIDATION \\ AND FACTORIAL INVARIANCE
}

\author{
GuAdALUPE DE LA IGlesia ${ }^{1,2}$, JUliana BEATRIZ STOVER ${ }^{1,3}$, \\ Alejandro CASTRo SOLANO ${ }^{1,2}$ Y MERCEDES FERNÁNDEZ-LIPORACE ${ }^{1,3}$
}

Cómo referenciar este artículo/How to reference this article:

de la Iglesia, G., Stover, J. B., Castro Solano, A. y Fernández-Liporace, M. (2015). Validez de constructo del LSB-50 en Adultos argentinos: validación cruzada e invarianza factorial [Construct validity of the LSB-50 in argentinean adults: cross-validation and factorial invariance]. Acción Psicológica, 12(2), 43-58. doi: http://dx.doi.org/10.5944/ap.12.2.15327

\section{Resumen}

El trabajo se propone aportar evidencias de validez referidas al Listado de Síntomas Breve (LSB-50; de Rivera y Abuín, 2012), instrumento psicométrico de screening para medir síntomas psicológicos, analizadas en una muestra de adultos argentinos. Los participantes fueron 1291 individuos distribuidos proporcionalmente según sexo (51\% mujeres; $49 \%$ varones) con edades entre 18 y 89 años $(M=38.9, D E=16.49)$. Se llevaron a cabo estudios de validación cruzada e invarianza factorial para examinar si un modelo de siete factores que se correspondía con siete escalas clínicas (Hipersensibilidad, Obsesiones-Compulsiones,

Correspondencia: Guadalupe de la Iglesia. Consejo Nacional de Investigaciones Científicas y Técnicas (CONICET); Universidad de Palermo, Argentina.

Email: gdelaiglesia@gmail.com

${ }^{1}$ Consejo Nacional de Investigaciones Científicas y Técnicas (CONICET).

${ }^{2}$ Universidad de Palermo, Argentina.

${ }^{3}$ Universidad de Buenos Aires, Argentina.

Recibido: 24 de septiembre de 2015

Aceptado: 25 de noviembre de 2015 
Ansiedad, Hostilidad, Somatización, Depresión, y Alteraciones del sueño) presentaba un ajuste adecuado $\mathrm{y}$ un funcionamiento similar en muestras diferenciadas según sexo y edad de los evaluados. Esta estructura heptafactorial exhibió un buen ajuste en las cuatro submuestras. Tras analizar simultáneamente el ajuste en las submuestras mencionadas a través de modelos jerárquicos en los que se impusieron distintas restricciones de igualdad, se arribó a resultados que indicaron la invarianza de las siete dimensiones del LSB-50. El cálculo de alfas ordinales indicó un buen nivel de consistencia interna para todas las escalas. Finalmente, las correlaciones con una medida externa de diagnóstico de psicopatología -el Personality Assessment Inventory (PAI; Morey, 2007)- indicaron, tal como se esperaba, una convergencia moderada. Se concluye que los análisis realizados proveen de contundentes evidencias de validez sobre los resultados arrojados por el LSB-50.

Palabras clave: screening; validez de constructo; invarianza factorial; validación cruzada.

\section{Abstract}

This paper aims at obtaining evidence of validity of the LSB-50 (De Rivera \& Abuín, 2012), a screening measure of psychopathology, in Argentinean adults. The sample consisted of 1291 individuals ( $49 \%$ males; $51 \%$ females) between 18 and 89 years-old $(M=38.9, S D=16.49)$. A crossvalidation study and factorial invariance studies were performed to test, in samples divided by sex and age, if a seven-factor structure that corresponds to seven clinical scales (Hypersensitivity, Obsessive-Compulsive, Anxiety, Hostility, Somatization, Depression, and Sleep disturbance) was adequate for the LSB-50. The seven-factor structure showed a good fit in all the subsamples. Next, the fit of the seven-factor structure was studied simultaneously in the aforementioned subsamples, through hierarchical models that imposed different constrains of equivalency. Results indicated the invariance of the seven clinical dimensions of the LSB-50. Ordinal alphas showed good internal consistency for all the scales. Finally, correlations with a diagnostic measure of psychopathology -el Personality Assessment Inventory (PAI; Morey, 2007)- indicated, as expected, moderate convergence. It is concluded that the analyses performed provide robust evidence of construct validity for the LSB-50.

Keywords: LSB-50; screening; construct validity; factorial invariance; cross-validation.

\section{Introducción}

Se estima que aproximadamente el $20 \%$ de la población argentina padece algún tipo de trastorno psicológico (Ministerio de Salud, 2010). Sin embargo, estos valores no son precisos debido a que han sido calculados a partir de proyecciones de estadísticas latinoamericanas y no de datos originados en Argentina. Una de las razones de esta vacancia en cuanto a datos robustos reside en la ausencia de herramientas de screening que cuenten con adecuadas evidencias de validez y confiabilidad para evaluar riesgo psicopatológico en población local.

Tal carencia en cuanto a instrumentos de cribado ha impedido la evaluación rápida de ciertas características psicológicas en la población local, impactando directamente en los objetivos centrales concernientes a la salud pública. De este modo, el desarrollo de tales escalas se constituye en un prerrequisito para establecer prevalencias psicopatológicas, analizar posibles factores a ellas asociados y detectar sujetos en riesgo de desarrollar un trastorno mental, de cara a la implementación de intervenciones preventivas tempranas o, eventualmente, atendiendo a la necesidad de profundizar en el diagnóstico detallado, específico y extensivo (Hernández Aguado et al., 2011; Kohn et al., 2005; Lewis, Sheringham, Kalim y Crayford, 2008). Así, este trabajo intenta abordar esta cuestión, teniendo como eje central la adaptación y análisis de propiedades psicométricas de una de tales herramientas, el Listado de Síntomas Breve-50 (De Rivera y Abuín, 2012) para su empleo en población local adulta. 
Tanto en Argentina como en el mundo, la herramienta de screening psicopatológico que más difusión ha obtenido es la versión revisada del Listado de 90 Síntomas, identificado como SCL-90-R por sus siglas en inglés (Derogatis, 1983). Debido a que su extensión fuera señalada como excesiva para realizar cribados en tiempos breves, el Brief Symptom Inventory (BSI; Derogatis 1975; Derogatis y Spencer, 1982) se propuso como una alternativa más viable. Dos décadas más tarde se publicó una versión aún más corta, el BSI-18 (Derogatis, 2001). A pesar de que la SCL-90-R continúa siendo habitualmente la más empleada, las otras dos también han sido adoptadas en los ámbitos de investigación y en el de aplicación (e.g., Aoian, Patsdaughter, Levin y Gianan, 1995; Torres, Miller y Moore, 2013; Zhang y Zhang, 2013).

La SCL-90-R ha sido objeto de varias críticas referidas a la inadecuada redacción de sus ítems, la cuestionable cobertura de la sintomatología que releva y, como ya se anticipara, su extensión. Aunque, su debilidad más saliente reside en la ausencia de evidencias rigurosas en cuanto a su validez factorial, dadas las dificultades que se han encontrado a la hora de replicar la estructura propuesta de nueve factores (e.g., Bados, Balaguer y Coronas, 2005; De Rivera y Abuín, 2012; Sandín, Valiente, Chorot, Santed y Lostao, 2008). A pesar de lo anterior, una de sus virtudes consiste en que ha mostrado capacidad para diferenciar pacientes de controles, obteniendo así evidencias de validez discriminante que resultan de interés a la hora de su uso en el ámbito aplicado (Holi, 2003).

A partir de las dificultades antes mentadas se desarrollaron otras versiones del instrumento. Davison et al. (1997), por ejemplo, diseñaron el Symptom Assessment-45 Questionnaire que, por su extensión, cuenta con la ventaja de tiempos más breves de administración. De todas maneras, a pesar de estas intenciones de mejora, algunos de los defectos de la escala de origen permanecieron en la nueva versión y sus adaptaciones (Alvarado, Sandín, Valdez-Medina, González-Arratia y Rivera, 2012; Sandín et al., 2008). De hecho, en todas las versiones antes nombradas los estudios factoriales brindaron resultados extremadamente intrincados y variables. Sus ítems presentaban cargas complejas y se agrupaban en estructuras diversas, desde unifactoriales, hasta bi, penta, hexa y octodimensionales (e.g., Abuín y de Rivera, 2014; Cyr, McKenna-Foley y Peacock, 1985; Daoud y Abojedi, 2010; De Las Cuevas et al., 1991; Hoffmann y Overall, 1978; Martínez Azumendi, Fernández Gómez y Beitía Fernández, 2001; Urbán et al., 2014), atribuyéndose estas diferencias a cuestiones de comorbilidad clínica (De Rivera y Abuín, 2012).

En este sentido, un hallazgo recurrente en estudios factoriales exploratorios (AFE) es una única dimensión superior usualmente identificada como una medida general de distrés psicológico (Benishek, Hayes, Bieschke y Stoffelmayr, 1998; Bonynge, 1993; Boulet y Boss, 1991; Cyr et al., 1985; Daoud y Abojedi, 2010; Grande, 2014; Loutsiou-Ladd, Panayiotou y Kokkinos, 2008; Martínez Azumendi, et al., 2001; Piersma, Boes y Reaume, 1994; Prunas, Sarno, Preti, Madeddu y Perugini, 2012; Torres et al., 2013; Zach, Toneatto y Streiner, 1998). En el caso de la SCL-90-R, por ejemplo, la constante falla en los intentos de replicar la estructura de nueve factores postulada complica la justificación del uso de las nueve puntuaciones correspondientes a las escalas. Así Bados et al. (2005) postularon el cálculo de una única medida de distress como la mejor opción. Sin embargo, a pesar de ser informativa, resolviendo las dificultades antes descriptas, tal medida general no da respuesta a las necesidades específicas de investigadores y clínicos.

Tampoco los estudios confirmatorios (AFC) han sido eficaces al momento de analizar la multidimensionalidad en el SCL-90-R, obteniéndose índices de ajuste inadecuados (Hardt, Gerbershagen y Franke, 2000; Ming-zhi, Heng-fen y Huei-fang, 2004; Rauter, Leonard y Swett, 1996; Schmitz et al., 2000; Vassend \& Skrondal, 1999) o apenas aceptables (Urbán et al., 2014). Tampoco el BSI logró valores que llegaran a los requeridos (Benishek et al., 1998; Liu, Chen, Cao y Jiao, 2013), sucediendo lo mismo con el SA-54 (Alvarado et al., 2012; Sandín et al., 2008).

De modo coherente con lo anterior, y tal como sucediera por ejemplo con la SCL-90-R, en los estudios de validación cruzada desarrollados, donde el ajuste del modelo se examina independientemente a través de distintos grupos segmentados según alguna característica en 
particular (e. g., sexo, edad), no lograron respaldar el modelo propuesto (Martínez Asumendi et al., 2001). Contrariamente a ello, Torres et al. (2013) informaron resultados satisfactorios para el BSI-18 al realizar estos análisis en una estructura unifactorial y en otra de tres factores en una muestra dividida según nacionalidad, el sexo y idioma de los participantes.

A la luz de lo anterior, las pruebas de invarianza factorial tampoco arribaron a conclusiones alentadoras sobre la equivalencia en el funcionamiento y ajuste del modelo comparando los distintos grupos pero, en este caso, de manera simultánea. En este marco metodológico, al momento no se ha logrado verificar la hipótesis de invarianza del modelo subyacente a la SCL-90-R en grupos segmentados según sexo (Vassend y Skrondal, 1999). Para el BSI, en cambio, la invarianza resultó verificada al analizar un grupo clínico y uno de población general, aunque no es un detalle menor que la estructura puesta a prueba fuera unidimensional (Daoud y Abojedi, 2010). No obstante, el análisis para adolescentes versus adultos no llegó a buenos resultados (Piersma et al., 1994). Torres et al. (2013) testearon la invarianza del BSI-18 según sexo, nacionalidad e idioma pero sólo la verificaron en el caso de las submuestras divididas teniendo en cuenta el sexo de los participantes.

El problema de la determinación de la consistencia interna merece un capítulo aparte, especialmente si se tiene en cuenta la arista metodológica. A pesar de que la mayoría de las medidas de screening psicopatológico utilizan opciones de respuesta con escalas Likert, la mayor parte de los análisis se realiza mediante índices alfas de Cronbach. Este procedimiento no es completamente adecuado a las propiedades de los datos, por lo que debiera calcularse una medida que tuviera en consideración la naturaleza ordinal de aquellos. Para tal propósito se propone el coeficiente alfa ordinal, basado en estimaciones obtenidas a través de matrices de correlación policórica (Elosúa y Zumbo, 2008), uso que en ningún caso fue puesto en práctica para la SCL-90-R y versiones subsiguientes. Al margen de ello, los resultados derivados del cálculo menos pertinente del alfa de Cronbach han informado, en líneas generales, una buena consistencia interna (e.g. Abuín y De Rivera, 2014; Caparrós Caparrós et al. 2007; Carrasco, Sánchez Moral, Ciccotelli y del
Barrio, 2003; Casullo y Castro Solano, 1999; Ruipérez, Ibáñez, Lorente, Moro y Ortet 2001).

Contemplando esta situación, que entraña gran cantidad de escollos teóricos y psicométricos que también repercuten en las prácticas de evaluación aplicada, De Rivera y Abuín (2012) desarrollaron recientemente el Listado de Síntomas Breve (LSB-50). Este test también está basado en la SCL-90-R, y pretende superar algunos de los defectos antes enunciados. La mejora contempló, por ejemplo, la exclusión de las escalas Psicoticismo e Ideación Paranoide, en virtud de la escasa claridad inherente a la formulación de sus ítems que producía dificultades para su comprensión. No obstante, la razón más importante residió en la noción de que tales síntomas pueden y deben ser fácilmente detectables en el curso de una entrevista clínica, por lo que resulta cuestionable e improcedente incluir su exploración en auto-reportes (Eaton, Neufeld, Chen y Cai, 2000). Adicionalmente, estudios factoriales han hallado que ambas escalas no emergen como dimensiones singulares (Prunas et al., 2012).

El diseño y adecuación de la LSB-50 también incluyó un ajuste lingüístico para lograr una mayor equivalencia en los términos a los modismos actuales del español, y la adición de una nueva escala clínica para la evaluación de síntomas relacionados con el sueño. Esta decisión resulta consistente con la quinta y última versión del Manual Diagnóstico y Estadístico de los Trastornos Mentales (American Psychiatric Association, 2013), en la que se hace hincapié en la prevalencia que los trastornos relacionados con el sueño exhiben, sumado a ello a su alta comorbilidad con otros síntomas psicopatológicos, cuestión ineludible en la práctica clínica.

El resultado fue una medida de 50 ítems, donde la severidad de los síntomas puede ser evaluada mediante siete escalas clínicas principales -Hipersensibilidad, Obsesiones-Compulsiones, Ansiedad, Hostilidad, Somatizaciones, Depresión, y Alteraciones del Sueño- y dos escalas clínicas adicionales - Psicorreactividad, que evalúa síntomas tanto Obsesiones-Compulsiones como Hipersensibilidad; y, Alteraciones del Sueño Extendida, que combina la evaluación de síntomas de ansiedad y depresión relacionados con el sueño -. Además se pueden cal- 
cular cuatro índices: (a) Índice de Severidad Global, (b) Número de Síntomas Presentes, (c) Intensidad de Síntomas Presentes, e (d) Índice de Riesgo Psicopatológico. Resulta de interés que la distorsión de respuesta se pueda analizar mediante las escalas de Magnificación y Minimización. Esto adquiere importancia a la luz del debate acerca de la precisión de la información que los auto-reportes permiten obtener. A pesar de estos cuestionamientos, se ha establecido que este formato de auto-informe resulta adecuado cuando el objetivo es el screening dado que su formato permite a los evaluados sentirse libres de informar sus síntomas con un bajo nivel de distorsión (Corcoran y Fischer, 2000; De Rivera y Abuín, 2012). Aunque no debe perderse de vista que se valora como ventajoso el empleo de escalas dirigidas a detectar estos patrones de respuesta con el fin de valorar la validez de cada protocolo, infrecuentemente se hallan incorporadas en este tipo de escalas de cribado por lo que su inclusión es un valor agregado.

Puesto que se trata de una medida relativamente nueva, al momento no se cuenta con muchas evidencias referidas a sus propiedades psicométricas en poblaciones diversas. Solamente ha sido analizada desde ese punto de vista en estudios españoles, colombianos y argentinos (Abuín y De Rivera, 2014; de la Iglesia, Stover, Fernández Liporace y Castro Solano, 2016; Rojas Gualdrón, 2012). Los resultados mostraron adecuados valores de consistencia interna -aunque calculada desde alfas de Cronbach y no ordinales- y un excelente ajuste en un estudio factorial confirmatorio de segundo orden para un modelo en el que las siete escalas clínicas principales cargaban en una medida general de distrés psicológico (de la Iglesia et al., en prensa). Por otra parte, obtuvo un buen ajuste en un AFC realizado en población colombiana (Rojas Gualdrón, 2012), aunque las dimensiones confirmadas no coincidieron con las propuestas por de los autores originales (De Rivera y Abuín, 2012).

Teniendo en cuenta las limitaciones de los inventarios antecedentes de la LSB-50, y con el fin de encarar un análisis más detallado de sus propiedades psicométricas, los objetivos del estudio consistieron en: (1) realizar un estudio de validación cruzada de la estructura de siete factores en cuatro submuestras analizadas independientemente (varones de 18 a 89 años, mujeres de 18 a 89 años, adultos de ambos sexos entre 18 y 34 años, y adultos de ambos sexos entre 35 y 89 años); (2) poner a prueba la invarianza factorial en grupos segmentados según sexo y edad, de modo de examinar si el funcionamiento y el ajuste del modelo resulta invariante en ambas submuestras a la vez; (3) estudiar la consistencia interna de cada escala mediante el cálculo de alfas ordinales; y (4) analizar las asociaciones de las escalas clínicas del LSB-50 con un criterio externo que mida psicopatología, el Personality Assessment Inventory (PAI; Morey, 2007). Cabe destacar que, a los fines de la brevedad, hasta aquí no se ha hecho alusión a los aspectos referidos a evidencias de validez externa de las escalas de screening con instrumentos de diagnóstico que permitan dar cuenta de la utilidad práctica de los primeros al momento de la detección de riesgo o de eventual pertinencia de especificar un diagnóstico. De esta manera, este aspecto también ha sido tomado en cuenta en el estudio aquí presentado.

\section{Método}

\section{Participantes}

La muestra estaba constituida por 1.291 adultos argentinos de población general, proporcionalmente distribuidos según sexo (51\% mujeres; $49 \%$ varones). La edad promedio era 38.90 años $(D E=16.49$, Mín $=$ 18, Máx = 89). En relación al estado civil, $46.7 \%$ informó estar casado o en una relación, $42.2 \%$ era soltero, $7.9 \%$ estaba divorciado/separado, y $3.1 \%$ era viudo/a. En cuanto a educación, 53.7\% había completado la escuela media, $40 \%$ había obtenido un título universitario, $6.1 \%$ había finalizado la escuela primaria, y el $0.2 \%$ reportó no haber tenido estudios formales. Para los estudios de validación cruzada e invarianza factorial se usaron, sucesivamente, como variables de segmentación el sexo y la edad. En primer lugar la muestra fue dividida en dos submuestras según sexo: una de mujeres $(n=633)$ y otra de varones $(n=$ 658). La edad promedio de las mujeres fue $39.01(D E=$ $16.83)$ y la edad promedio de los varones era $38.79(D E$ =16.16). En segundo lugar, nuevamente a partir de la 
muestra principal se segmentaron dos grupos de edad: uno de 18 a 34 años $(n=669,51 \%$ varones, $49 \%$ mujeres) y otro de 35 a 89 años $(n=622,51 \%$ varones, $49 \%$ mujeres).

\section{Materiales}

Listado de Sintomas Breve (LSB-50; De Rivera y Abuín, 2012; de la Iglesia et al., en prensa). Es una escala de 50 ítems que mide diferente síntomas psicológicos mediante siete escalas clínicas principales: (1) Hipersensibilidad (siete ítems), se refiere a la sensibilidad tanto intra como interpersonal (e.g., "Me parece que otras personas me observan o hablan de mí"); (2) Obsesiones-Compulsiones (siete ítems), apunta a cubrir la presencia de dudas, rituales y compulsiones (e.g., "Tengo que hacer las cosas muy despacio para estar seguro de que las hago bien"); (3) nsiedad (nueve ítems), indaga acerca de síntomas de pánico, trastorno de ansiedad generalizada y fobias (e.g., "Siento miedo en la calle o en espacios abiertos"); (4) Hostilidad (seis ítems), interroga sobre comportamientos vinculados a la ira, enojo y resentimiento (e.g., "Tengo ganas de romper o destruir algo"); (5) Somatizaciones (ocho ítems), evalúa síntomas somáticos que tienen base en problemas psicológicos o médicos (e.g., "Mi corazón palpita o va muy deprisa"); (6) Depresión (diez ítems), examina síntomas relacionados con escasa energía, culpa, tristeza y desesperanza (e.g., "Me siento triste"); y (7) Alteraciones del sueño (tres ítems), examina posibles dificultades de sueño desde la perspectiva del bienestar (e.g., "Me despierto de madrugada"). Los ítems se responden mediante una escala Likert de cinco posiciones que varía desde $0=n a d a$ hasta $4=$ mucho . En el estudio llevado a cabo por de la Iglesia et al. (2015) los alfas de Cronbach para las escalas clínicas en población de adultos argentinos fueron: Hipersensibilidad $=.79$, Obsesiones-Compulsiones $=$. 72 , Ansiedad $=.83$, Hostilidad $=.81$, Somatización $=$ .77 , Depresión $=.82$, y Alteraciones del sueño $=.83$.

Personality Assessment Inventory -Inventario de Evaluación de la Personalidad- (PAI; Morey, 2007; Ortiz-Tallo, Santamaría, Cardenal y Sánchez, 2011; Stover,
Castro Solano y Fernández Liporace, 2015). Este instrumento fue diseñado para evaluar personalidad psicopatológica en adultos. Está compuesto por 344 ítems que permiten reportan en el cálculo de diversas escalas. La respuesta consiste en una escala Likert de cuatro posiciones variante entre $0=$ falso y $3=$ completamente verdadero. Para esta investigación sólo se tuvieron en cuenta las siguientes escalas y subescalas: Paranoia, ObsesivoCompulsivo (subescala de Trastornos Relacionados con la Ansiedad), Ansiedad, Agresión, Quejas Somáticas, Depresión y Estrés. La adaptación local incluyó el análisis de consistencia interna mediante alfas de Cronbach y análisis de componentes principales para estudiar su dimensionalidad (Stover et al., en prensa). En ese estudio los alfas de Cronbach calculados en adultos argentinos para las escalas aquí analizadas fueron Paranoia $=.78$, Obsesivo-Compulsivo $=.61$, Ansiedad $=.86$, Agresión $=$ .82 , Quejas Somáticas $=.84$, Depresión $=.82$ y Estrés $=$ .60 .

\section{Procedimiento}

Se trabajó con un muestreo no probabilístico, con participación voluntaria. Todos los participantes firmaron un consentimiento informado en el que se explicaba en detalle el objetivo de la investigación y se aseguraba la confidencialidad y anonimato de los datos personales y resultados, así como la opción de cesar la respuesta en cualquier momento. La recolección de datos estuvo a cargo de estudiantes avanzados de psicología, y su trabajo fue supervisado por investigadores formados que además eran psicólogos habilitados y altamente entrenados en tareas de evaluación. En todos los casos los protocolos se administraron individualmente con especial cuidado de las condiciones de evaluación, que fueron igualadas para mantener constantes los procedimientos, en pos de asegurar la comprensión de la consigna y el apego al entrenamiento recibido por los aplicadores de la escala, así como la calidad de la respuesta y el bienestar de los participantes. 


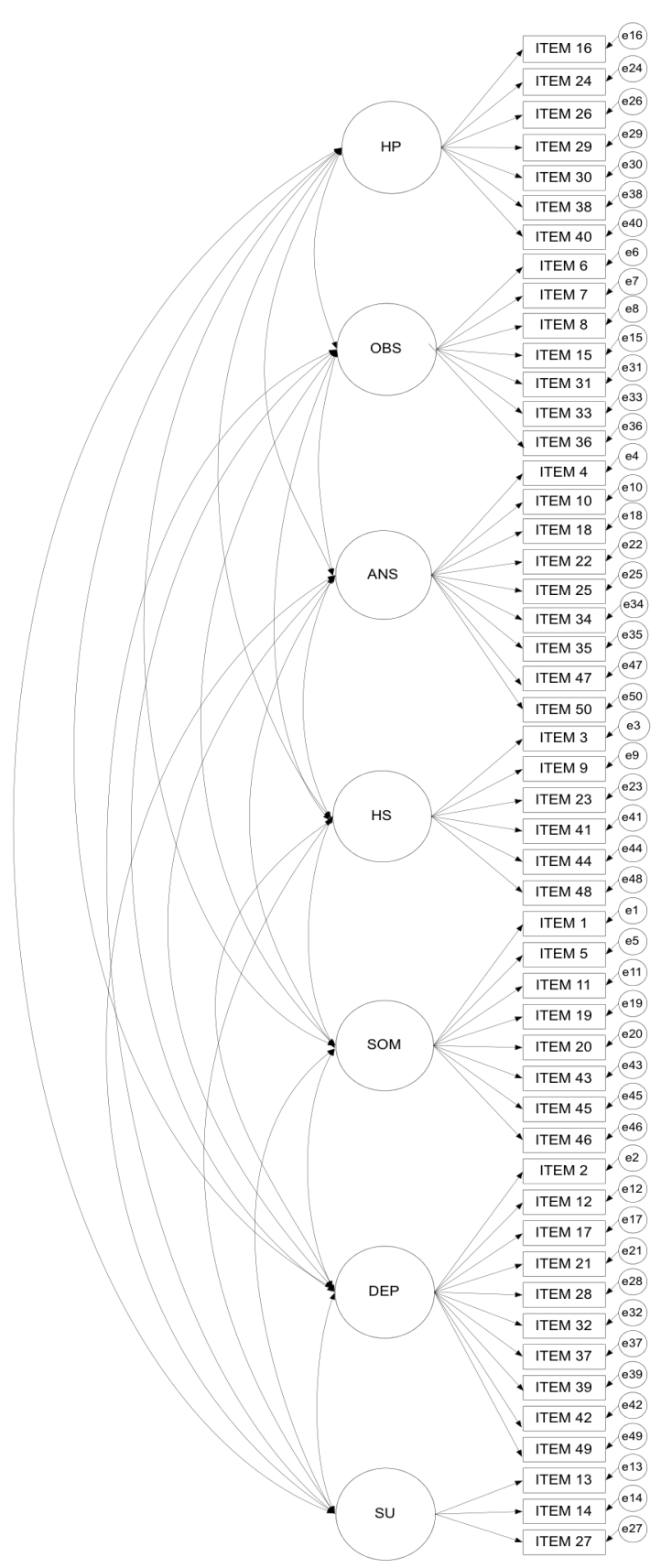

Figura 1. Modelo de medida analizado. HP: Hipersensibilidad; OBS: Obsesiones-Compulsiones; ANS: Ansiedad; HS: Hostilidad; SOM: Somatizaciones; DEP: Depresión; SU: Alteraciones del sueño

El modelo de medida puesto a prueba proponía una estructura factorial de primer orden en la que 50 elementos observables (síntomas cargaban en siete escalas clínicas (ver Figura 1). Para realizar los estudios de validación cruzada e invarianza factorial se eligió el software EQS 6.2. 
Tabla 1

Validación cruzada: Ajuste del modelo según sexo y edad

\begin{tabular}{lllll}
\hline & CFI & NFI & IFI & RMSEA (IC 90\%) \\
\hline Sexo & & & & .982 \\
Varones & .982 & .960 & .984 & $.035(.033-.037)$ \\
Mujeres & .984 & .966 & & $.036(.033-.038)$ \\
& & & & \\
Edad & & .961 & .981 & $.037(.035-.040)$ \\
18 a 34 años & .981 & .961 & $.035(.033-.038)$ \\
35 a 89 años & .985 & .967 & .985 & \\
\hline
\end{tabular}

En ambos casos el método de estimación fue máxima verosimilitud robusto empleando una matriz de correlaciones policóricas debido a la naturaleza ordinal de los ítems (escalas Likert). Estos tipos de método y matriz son más apropiados para variables ordinales cuando existe evidencia de valores altos de asimetría y curtosis (Freiberg Hoffmann, Stover, de la Iglesia y Fernández Liporace, 2013; Muthén y Kaplan, 1985).

Para evaluar el ajuste del modelo en el estudio de validación cruzada se analizaron diversos índices de ajuste obtenidos con el método de estimación robusto: CFI (Comparative Fit Index), NFI (Normed Fit Index), IFI (Incremental Fit Index) y RMSEA (Root Mean Square Error of Approximation). CFI, NFI y IFI fueron considerados apropiados si se encontraban por encima de .90. El valor del RMSEA, por otro lado, debía ser inferior a .05 (Byrne, 2006; Kline, 1998).

Con el fin de analizar la invarianza factorial se establecieron modelos jerárquicos con restricciones progresivas. El MODELO 1 no imponía restricciones de igualdad, en el MODELO 2 se impuso la restricción de igualdad de cargas factoriales y en el MODELO 3, la igualdad de cargas y covarianzas. Este análisis fue realizado dos veces; primero, para examinar la invarianza en la muestra de mujeres y varones y segundo, para exami- nar la invarianza en el grupo de adultos más jóvenes y adultos medios y mayores (18 a 34 años vs. 35 a 89 años). Siguiendo a Byrne (2006), para evaluar el ajuste se estudiaron los valores del estadístico Satorra Bentler (SB), el CFI y el RMSEA. La hipótesis de invarianza de verificó mediante el análisis el $\Delta \mathrm{S}-\mathrm{B}$ y el $\Delta \mathrm{CFI}$. En el primer caso, se esperaba que la diferencia no fuera estadísticamente significativa en un nivel de alfa de $.05 \mathrm{y}$, en el caso del CFI el delta no debía superar el valor de .01.

Finalmente, para obtener evidencias de validez externa se calcularon correlaciones con escalas del PAI por medio del paquete SPSS 18.0, en tanto que los alfas ordinales fueron estimados con Excel, de acuerdo a las directrices de Elosúa y Zumbo (2008).

\section{Resultados}

Los índices de ajuste obtenidos para la submuestra de mujeres y la de varones en el estudio de validación cruzada indicaron un excelente ajuste del modelo de siete factores en ambos grupos (ver tabla 1). El ajuste también fue adecuado cuando el modelo de medida se puso a prueba en las dos submuestras segmentadas según el corte de edad en 35 años.

Tabla 2

Invarianza factorial en submuestras conformadas según sexo (varones vs. mujeres)

\begin{tabular}{|c|c|c|c|c|c|c|c|c|c|}
\hline & $\begin{array}{l}\text { Satorra- } \\
\text { Bentler }\end{array}$ & $g l$ & CFI & RMSEA (IC90\%) & $\begin{array}{l}\text { Comparación } \\
\text { de modelos }\end{array}$ & $\Delta$ S-B & $\Delta g l$ & $p$ & $\Delta \mathrm{CFI}$ \\
\hline $\begin{array}{l}\text { Modelo } 1 \\
\text { Sin restricciones }\end{array}$ & 4185.44 & 2308 & .983 & $.036(.034-.037)$ & 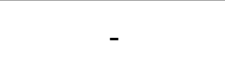 & - & - & - & - \\
\hline $\begin{array}{l}\text { Modelo } 2 \\
\text { Restricción de cargas } \\
\text { Modelo } 3\end{array}$ & 4251.16 & 2351 & .983 & $.035(.034-.037)$ & 2 vs 1 & 62.08 & 43 & .029 & 0 \\
\hline $\begin{array}{l}\text { Restricción de cargas } \\
\text { y covarianzas }\end{array}$ & 4279.11 & 2372 & .983 & $.035(.034-.037)$ & 3 vs 1 & 80.67 & 64 & .077 & 0 \\
\hline
\end{tabular}


Tabla 3

Invariancia factorial en submuestras conformadas según edad (18 a 34 años vs. 35 a 89 años)

\begin{tabular}{|c|c|c|c|c|c|c|c|c|c|}
\hline & Satorra-Bentler & $g l$ & CFI & RMSEA (IC90\%) & $\begin{array}{l}\text { Comparación } \\
\text { de modelos }\end{array}$ & $\Delta$ S-B & $\Delta g l$ & $p$ & $\triangle \mathrm{CFI}$ \\
\hline $\begin{array}{l}\text { Modelo } 1 \\
\text { Sin restricciones }\end{array}$ & 4281.30 & 2308 & .983 & $.036(.035-.038)$ & - & - & - & - & - \\
\hline $\begin{array}{l}\text { Modelo } 2 \\
\text { Restricción de cargas } \\
\text { Modelo } 3\end{array}$ & 4359.59 & 2351 & .983 & $.036(.035-.038)$ & 2 vs 1 & 74.18 & 43 & .002 & 0 \\
\hline $\begin{array}{l}\text { Restricción de cargas } \\
\text { y covarianzas }\end{array}$ & 4393.94 & 2372 & .983 & $.036(.035-.038)$ & 3 vs 1 & 106.95 & 64 & $<.001$ & 0 \\
\hline
\end{tabular}

Asimismo y como se comentara en el apartado previo, se calcularon modelos jerárquicos con restricciones progresivas para examinar la invarianza factorial, en las submuestras de varones y mujeres, y a continuación en la de adultos jóvenes y mayores.

Tanto para el MODELO 1, como para el 2 y el 3, los valores del CFI como del RMSEA fueron adecuados (ver Tabla 2). En la tabla 2 también puede observarse la comparación de modelos. La diferencia en el estadístico Satorra-Bentler $(\triangle \mathrm{S}-\mathrm{B})$ al compararse el MODELO 1 con el MODELO 2 resultó estadísticamente significativa considerando un nivel de alfa de .05 (no así en uno de $.01)$. No se verificó significación estadística al compararse el MODELO 1 con el MODELO 3. Adicionalmente, en todos los casos el $\Delta$ CFI fue igual a cero.

Se utilizaron los mismos procedimientos para testear la invarianza factorial en los dos grupos de edad. El ajuste evaluado mediante el CFI y el RMSEA fue adecuado en todos los casos (Tabla 3). En la comparación de modelos, la diferencia en el estadístico SatorraBentler fue estadísticamente significativa entre los MODELOS 1 y 2, así como también entre los MODELO 1 y 3. En ningún caso se registraron cambios en el valor del CFI (Tabla 3).

Tabla 4

Alfas ordinales para las escalas clínicas
Para el cálculo de los alfas ordinales se trabajó con las cargas factoriales obtenidas mediante el análisis de la matriz policórica, y siguiendo las recomendaciones planteadas por Elosúa y Zumbo (2008). Tales coeficientes indicaron una excelente consistencia interna (Tabla 4).

Para finalizar se calcularon correlaciones de Pearson entre las puntuaciones de las escalas clínicas del LSB-50 y las escalas del PAI que desde el punto de vista descriptivo y teórico resultaban pertinentes. La asociación entre Hipersensibilidad y Paranoia fue positiva y débil ( $r$ $=.36, p<.001)$. Lo mismo ocurrió al con la correlación entre la escala Obsesiones-Compulsiones del LSB-50 y la subescala Obsesivo-Compulsivo del PAI $(r=.32, p<$ .001). Las escalas de Ansiedad de ambos tests se asociaron de manera positiva y moderada $(r=.61, \mathrm{p}<.001)$. La correlación entre la escala Hostilidad y la escala Agresión del PAI fue positiva y moderada $(r=.54, \mathrm{p}<.001)$. La asociación entre la escala Somatización del LSB-50 y Quejas Somáticas del PAI fue positiva y moderada $(r=$ $.55, \mathrm{p}<.001)$. Las escalas de Depresión se asociaron de manera positiva y moderada $(r=.61, \mathrm{p}<.001)$. Finalmente, la correlación con la escala de Estrés del PAI, que había sido elegida como el criterio externo para Alteraciones del Sueño, resultó positiva y débil $(r=.24, \mathrm{p}<$ $.001)$.

\begin{tabular}{lc}
\hline & Alfa ordinal \\
\hline Hipersensibilidad & .85 \\
Obsesiones-Compulsiones & .78 \\
Ansiedad & .90 \\
Hostilidad & .88 \\
Somatizaciones & .84 \\
Depresión & .89 \\
Alternaciones del sueño & .88 \\
\hline
\end{tabular}




\section{Discusión}

El objetivo principal de este trabajo consistió en aportar evidencias adicionales referidas a las propiedades psicométricas del LSB-50 (De Rivera y Abuín, 2012) en una muestra de adultos argentinos. Al momento, la investigación abocada a establecer tales propiedades psicométricas de otras medidas de screening psicopatológico como la SCL-90, la SCL-90-R, el BSI, el BSI-18 y el SA-54 se ha topado con diversas dificultades. Entre ellas, la principal ha sido la obtención de evidencias de validez de constructo de un modelo multidimensional replicable que brindara el soporte necesario para fundamentar el cálculo de puntuaciones independientes para las escalas clínicas (e.g., Benishek et al., 1998; Hardt et al., 2000; Liu et al., 2013; Ming-zhi et al. 2004; Rauter et al., 1996; Sandín et al., 2008; Schmitz et al., 2000; Vassend y Skrondal, 1999). Es por ello que cualquier instrumento que alegue ser una mejora de aquellos mencionados debería contar necesariamente con rigurosas evidencias en cuanto a su dimensionalidad. En este sentido los estudios desarrollados, de validación cruzada e invarianza factorial, se encuentran entre los procedimientos más estrictos para examinar si el ajuste del modelo se mantiene en muestras diferenciadas. En el primer caso, analizando el ajuste de aquellas de manera independiente. En el segundo, en cambio, estudiando el ajuste de modo simultáneo con todas las submuestras de interés, y estableciendo sucesivas restricciones de equivalencias de parámetros (cargas y covarianzas, por ejemplo) para poner a prueba la hipótesis de invarianza.

En el caso de la validación cruzada, los índices de ajuste obtenidos en las cuatro submuestras aisladas de acuerdo a sexo y edad fueron adecuados y otorgan sustento a la estructura heptafactorial propuesta.

Además, se encontraron evidencias de invarianza factorial cuando se impusieron distintas restricciones de equivalencias. A pesar de que el $\Delta \mathrm{S}$-B resultó estadísticamente significativo cuando se analizó la invarianza en las muestras divididas de acuerdo con la edad, el $\Delta$ CFI resultó ser nulo y el ajuste del modelo fue bueno (Byrne, 2006). Es importante destacar que los análisis aquí desarrollados fueron realizados al nivel del ítem. Esto provee de evidencias de validez de constructo mucho más estrictas que aquellas obtenidas cuando las sumatorias de los ítems son el input en la factorización.

Es posible que la justificación de estos resultados alentadores resida en dos aspectos. Primero, tal como fuera mencionado, el LSB-50 fue cuidadosamente diseñado para superar aquellas dificultades encontradas en la SCL-90-R. Es posible que el estudio minucioso del instrumento de origen, sumado a la consideración de resultados de investigaciones precedentes haya impactado positivamente en la validez de contenido, y en consecuencia, esto haya afectado favorablemente los resultados de los estudios factoriales aquí presentados. En segundo lugar, los análisis aquí efectuados tomaron en consideración la naturaleza ordinal de los elementos componentes del test. Es por ello que se utilizaron tanto matrices policóricas cuanto métodos robustos en todos los casos. Así, es factible que esta decisión estadística haya favorecido los resultados aquí obtenidos.

Otro análisis relacionado con este último aspecto fue el cálculo de los alfas ordinales. Tal como fuera señalado, resultados precedentes informados para otros instrumentos similares al LSB-50 indicaban, en general, buenos resultados en términos de consistencia interna (e.g., Abuín y De Rivera, 2014; Caparrós Caparrós et al. 2007; Carrasco et al., 2003; Casullo y Castro Solano, 1999; Ruipérez et al., 2001). Sin embargo, considerando que el alfa ordinal ha sido propuesto como una mejor opción cuando los valores obtenidos provienen de escalas Likert (Elosúa y Zumbo, 2008), su cálculo parecía más apropiado. Tal es así que los hallazgos indican una mejora en comparación con los valores previamente obtenidos para los alfa de Cronbach.

Finalmente, era necesario obtener evidencias acerca de la validez externa del test. Para ello, se seleccionaron algunas escalas del PAI (Morey, 2007) el cual funcionó como criterio externo de diagnóstico psicopatológico. Las correlaciones de Pearson indicaron, tal como era esperado, que la mayoría de las asociaciones eran positivas y moderadas. Las excepciones fueron Hipersensibilidad, Obsesiones-Compulsiones y Alteraciones del sueño que tuvieron correlaciones positivas pero débiles con Paranoia, Obsesivo-Compulsivo y Estrés, respectivamente. 
En el caso de Hipersensibilidad, la asociación débil con Paranoia puede deberse a los síntomas que abarcan. Por ejemplo, se espera que alguien con un diagnóstico de paranoia presente también hipersensibilidad. Sin embargo, lo opuesto puedo no ser necesariamente verdadero. Es decir, alguien con síntomas de hipersensibilidad no forzosamente cumplirá con todos los criterios para arribar a un diagnóstico de paranoia.

Algo similar ocurre en relación a las medidas de obsesiones-compulsiones. La escala Obsesiones-Compulsiones del LSB-50 se refiere a la presencia de rituales o compulsiones y dudas. Por otro lado, la escala Obsesivo-Compulsivo del PAI alude mayormente a perfeccionismo, rigidez e indecisión. Ambas comparten el componente de dudas/indecisión, pero discrepan en el resto de los síntomas incluidos. Esta diferencia puede explicar la correlación positiva pero débil entre ambas.

La escala Estrés del PAI fue elegida como el criterio externo para Alteraciones del Sueño de LSB-50, debido a que el primer inventario no cuenta con una escala correspondiente más equiparable a la segunda en cuanto a contenido. Claramente ambas medidas no evalúan el mismo constructo. Debido a que, sustentando la elección del criterio externo subyacía la hipótesis de que un individuo afectado por estrés debiera experimentar algún nivel de alteración en su dormir, se esperaba una correlación positiva entre ellas. Y puesto que tal fue el resultado obtenido, ello da sustento a esa presunción pese la debilidad del índice hallado.

Debe destacarse de todos modos que el criterio utilizado para la interpretación de las correlaciones fue eminentemente estadístico (Hernández Sampieri, Fernández Collado y Baptista Lucio, 2010) Así, una correlación que, desde este punto de vista puede valorarse como débil o moderada, desde la arista clínica podría estar indicando que la asociación sintomatológica reviste el suficiente interés como para pasar a una instancia de diagnóstico ante la detección de riesgo en el screening. Ello se vuelve aún más importante si se tienen en cuenta los comentarios precedentes sobre la diversidad sintomatológica aludida por las escalas que se están comparando, donde el LSB-50 es de cribado y, por ende, debe ser altamente sensible para detectar grados leves e in- cluso poco perceptibles de distrés psicológico que pueden pasar desapercibidos en la observación y entrevistas.

Dos aspectos surgen al considerarse las limitaciones de la investigación. Primero, el muestreo fue no probabilístico. A pesar de ser una práctica usual en la investigación en psicología, debe considerarse que las muestras no probabilísticas conllevan restricciones de cara a la generalización de los resultados obtenidos. En segundo lugar, al momento, el LSB-50 cuenta ahora con nuevas, aunque acotadas evidencias de validez externa. Futuras investigaciones deberían continuar analizando este aspecto y proveer información acerca de su sensibilidad, especificidad, valor predictivo positivo y negativo, así como el cálculo de curvas ROC para establecer valores de corte adecuados.

Para concluir, se han obtenido contundentes evidencias de validez factorial y de confiabilidad de la adaptación argentina del LSB-50. Debe destacarse que los resultados obtenidos dan soporte al cálculo de siete escalas clínicas: Hipersensibilidad, ObsesionesCompulsiones, Ansiedad, Hostilidad, Somatizaciones, Depresión y Alteraciones del sueño. Es por ello que es utilizable tanto en los campos de investigación como los de aplicación con población de adultos argentinos. Ulteriores desarrollos se encargarán de ampliar y profundizar estos estudios 


\section{Referencias}

Abuín, M. R. y de Rivera, L. (2014). La medición de síntomas psicológicos y psicosomáticos: El Listado de Síntomas Breve (LSB-50). Clínica y Salud, 25, 131-141.

http://dx.doi.org/10.1016/j.clysa.2014.06.001

American Psychiatric Association. (2013). Diagnostic and statistical manual of mental disorders ( $5^{\mathrm{a}} \mathrm{Ed}$.). Arlington, VA: Autor.

Aoian, K. J., Patsdaughter, C. A., Levin, A. y Gianan, M. E. (1995). Use of the Brief Symptom Inventory to assess psychological distress in three immigrants groups. International Journal of Social Psychology, 41, 31-46.

Alvarado, B. G., Sandín, B., Valdez-Medina, J. L., González-Arratia, N. y Rivera, S. (2012). Análisis factorial confirmatorio del Cuestionario SA-45 en una muestra mexicana. Anales de Psicología, 28(2), 426-433.

doi:

http://dx.doi.org/10.6018/analesps.28.2.148851

Bados, A., Balaguer, G. y Coronas, M. (2005). Qué mide realmente el SCL 90 R?: Estructura factorial en una muestra mixta de universitarios y pacientes. Psicología Conductual, 13(2), 181-196.

Benishek, L. A., Hayes, C. M., Bieschke, K. J. y Stoffelmayr, B. E. (1998). Exploratory and confirmatory analyses of the Brief Symptom Inventory among substance abusers. Journal of Substance Abuse, 10, 103-114. doi: http://dx.doi.org/10.1016/S08993289(99)80127-8

Bonynge, E. R. (1993). Unidimensionality of SCL-90-R scales in adult and adolescent crisis samples. Journal of Clinical Psychology, 49, 212-215. doi: http://dx.doi.org/ 10.1002/10974679(199303)49:23.0.CO

Boulet, J. y Boss, M. W. (1991). Reliability and validity of the Brief Symptom Inventory. Psychological
Assessment, $\quad 3, \quad 433-437 . \quad$ doi: http://dx.doi.org/10.1037/1040-3590.3.3.433

Byrne, B. M. (2006). Structural Equation Modeling with EQS. Basic concepts, applications and programming $\left(2^{\mathrm{a}} \mathrm{Ed}\right)$. New York: Routledge.

Caparrós Caparrós, B., Villar Hoz, E., Juan Ferrer, J. y Viñas Poch, F. (2007). Symptom Check-List-90-R: Fiabilidad, datos normativos y estructura factorial en estudiantes. International Journal of Clinical and Health Psychology, 7(3), 781-794.

Carrasco, M. A., Sánchez Moral, V., Ciccotelli, H. y del Barrio, V. (2003). Listado De Síntomas SCL-90-R: Análisis de su Comportamiento en una Muestra Clínica. Acción Psicológica, 2(2), 149-161.

Casullo, M. M. y Castro Solano, A. (1999). Síntomas psicopatológicos en estudiantes adolescentes argentinos. Aportaciones del SCL-90. Anuario de Investigaciones, 7, 147-157.

Corcoran, K. y Fischer, J. (2000). Measures for Clinical Practice. A sourcebook. New York: The Free Press.

Cyr, J. J., McKenna-Foley, J. M. y Peacock, E. (1985). Factor structure of the SCL-90-R: Is there one? Journal of Personality Assessment, 49(6), 571-578. doi: http://dx.doi.org/10.1207/s15327752jpa4906_2

Daoud, F. S. y Abojedi, A. A. (2010). Equivalent factorial structure of the Brief Symptom Inventory (BSI) in clinical and nonclinical Jordanian populations. European Journal of Psychological Assessment, 26(2), 116-121. doi: http://dx.doi.org/10.1027/10155759/a000016)

Davison, M. K., Bershadsky, B., Bieber, J., Silversmith, D., Maruish, M. E. y Kane, R. L. (1997). Development of a brief, multidimensional, self-report instrument for treatment outcomes assessment in psychiatric settings: Preliminary findings. Assessment, 4, 259-276. 
de la Iglesia, G., Stover, J. B., Fernández Liporace, M. y Castro Solano, A. (en prensa). Listado de Síntomas Breve (LSB-50) -Short Checklist of Symptoms- in Argentinean adults: Psychometric study of its main clinical scales. Research in Psychotherapy: Psychopathology, Process and Outcome.

De las Cuevas, C., González De Rivera, J. L., Henry Benítez, M., Monterrey, A. L., Rodríguez-Pulido, F. y Gracia Marco, R. (1991). Análisis factorial de la versión española del SCL-90-R en la población general. Anales de Psiquiatría, 7(3), 93-96.

De Rivera, L. y Abuín, M. L. (2012). LSB-50 Listado de Sintomas Breve: Manual. España: TEA.

Derogatis, L. R. (1975). Brief Symptom Inventory. Baltimore: Clinical Psychometric Research.

Derogatis, L. R. (1983). SCL-90-R: Administration, scoring, and procedures manual II. Baltimore: Clinical Psychometric Research.

Derogatis, L R. (2001). The Brief Symptom Inventory 18 (BSI 18). Administration, scoring and procedures manual. Minneapolis: NCS Pearson.

Derogatis, L. R. y Spencer, M. S. (1982). The Brief Symptom Inventory (BSI): Administration, scoring, and procedures manual. Baltimore: Johns Hopkins University School of Medicine, Clinical Psychometrics Research Unit.

Eaton, W. W., Neufeld, K., Chen, L. y Cai, G. (2000). A comparison of self-report and clinical diagnostic interviews for depression: Diagnostic Interview Schedule and Schedules for Clinical assessment in Neuropsychiatry in the Baltimore Epidemiologic Catchment area follow-up. Archives of General Psychiatry, 57(3), 217-222. doi: http://dx.doi.org/10.1001/archpsyc.57.3.217

Elosúa, P. y Zumbo, B. D. (2008). Coeficientes de fiabilidad para escalas de respuesta categórica ordenada. Psicothema, 20(4), 896-901.
Freiberg Hoffmann, A., Stover, J. B., de la Iglesia, G. y Fernández Liporace, M. (2013). Correlaciones Policóricas y Tetracóricas en Estudios Exploratorios y Confirmatorios. Ciencias Psicológicas, 7(2), 151-164.

Grande, T. L. (2014). Path analysis of the SCL-90-R: Exploring use in outpatient assessment. Measurement and Evaluation in Counseling and Development. Advance online publication. doi: http://dx.doi.org/ $10.1177 / 0748175614538061$.

Hardt, J., Gerbershagen, H. U. y Franke, P. (2000). The symptom checklist, SCL-90-R: Its use and characteristics in chronic pain patients. European Journal of Pain, 4(2), 137-148. doi: http://dx.doi.org/10.1053/eujp.2000.0162

Hernández Aguado, I., Gilde Miguel, A., Delgado Rodríguez, M., Bolúmar Montrull, F., Benavides, F. G., Porta Serra, M. y Lumbrera Lacarra, B. (2011). Manual de Epidemiología y Salud Pública para grados en Ciencias de la Salud. Madrid, España: Médica Panamericana.

Hernández Sampieri, R., Fernández Collado, C. y Baptista Lucio, M. P. (2010). Metodología de la investigación. México: McGraw Hill.

Hoffmann, N.G. y Overall, P.B. (1978). Factor Structure of the SCL-90 in a Psychiatric Population. Journal of Consulting and Clinical Psychology, 46(6), 11871191. doi: http://dx.doi.org/10.1037/0022006X.46.6.1187

Holi, M. (2003). Assessment of psychiatric symptoms using the SCL-90 (Tesis de doctorado, Department of Psychiatry, University of Helsinki, Helsinki, Finland). Recuperado de http://ethesis.helsinki.fi/julkaisut/laa/kliin/vk/holi/asse ssme.pdf

Kline, R. B. (1998). Principles and practice of structural equation modeling. New York: The Guilford Press.

Kohn, R., Levav, I., Caldas de Almeida, J. M., Vicente, B., Andrade, L., Caraveo-Anduaga, J. J. y Saraceno, 
B. (2005). Los trastornos mentales en América Latina y el Caribe: Asunto prioritario para la salud pública. Revista Panamericana de Salud Pública, 18(4/5), 229-240.

Lewis, G., Sheringham, J., Kalim, K. y Crayford, T. (2008). Mastering Public Health: A postgraduate guide to examinations and revalidation: A Guide to Examinations and Revalidation. Londres, UK: Royal Society of Medicine Press.

Liu, Z., Chen, H., Cao, B. y Jiao, F. (2013). Reliability and validity of Chinese version of Brief Symptom Inventory in high school students. Chinese Journal of Clinical Psychology, 21(1), 32-34.

Loutsiou-Ladd, A., Panayiotou, G. y Kokkinos, C. M. (2008). A review of the factorial structure of the Brief Symptom Inventory (BSI): Greek evidence. International Journal of Testing, 8(1), 90-110. doi: http://dx.doi.org/10.1080/15305050701808680

Martínez Azumendi, O., Fernández Gómez, C. y Beitía Fernández, M. (2001). Variabilidad factorial del SCL-90-R en una muestra psiquiátrica ambulatoria. Actas Españolas de Psiquiatría, 29(2), 95-102.

Ministerio de Salud (2010). Estimación de la población afectada de 15 años y más por trastornos mentales y adicciones. Recuperado

de http://www.inclusionmental.com.ar/contents/bibliotec a/1329413814_-estimacion-de-la-poblacionafectada-por-salud-mental-arg.pdf

Ming-zhi, X., Heng-fen, L. y Huei-fang, Z. (2004). Factorial Structure of the Symptom Checklist 90 (SCL-90) in College Students. Chinese Journal of Clinical Psychology, 12(4), 2004, 348-351.

Morey, L. (2007). Personality Assessment Inventory (PAI). Professional Manual (2 ${ }^{\circ}$ Edition). Florida: Psychological Assessment Resources.

Muthén, B. y Kaplan, D. (1985). A comparison of some methodologies for the factor analysis of non-normal Likert variables. British Journal of Mathematical and Statistical Psychology, 38, 171-189.
Ortiz-Tallo, M., Santamaría, P., Cardenal, V. y Sánchez, M.P. (2011). Inventario de Evaluación de la Personalidad. Madrid, España: TEA.

Piersma, H. L., Boes, J. L. y Reaume, W. M. (1994). Unidimensionality of the Brief Symptom Inventory (BSI) in adult and adolescent inpatients. Journal of Personality Assessment, 63(2), 338-344. doi: http://dx.doi.org/10.1207/s15327752jpa6302_12

Prunas, A., Sarno, I., Preti, E., Madeddu, F. y Perugini, M. (2012). Psychometric properties of the Italian version of the SCL-90-R: A study on a large community sample. European Psychiatry, 27(8), 591597. doi: http://dx.doi.org/10.1016/j.eurpsy.2010.12.006

Rauter, U. K., Leonard, C. E. y Swett, C. P. (1996). SCL90-R factor structure in an acute, involuntary, adult psychiatric inpatient sample. Journal of Clinical Psychology, 52(6), 625-629. doi: http://dx.doi.org/10.1002/(SICI)10974679(199611)52:63.3.CO

Rojas Gualdrón, D. F. (2012, agosto). Capacidad Explicativa de los Síntomas del LSB-50 sobre un Único Factor de Psicopatología General. Trabajo presentado en el I Congreso Internacional de Psicología: Investigación y Responsabilidad Social, Bucaramanga, Colombia.

Ruipérez, M. A., Ibáñez, M. I., Lorente, E., Moro, M. y Ortet, G. (2001). Psychometric Properties of the Spanish Version of the BSI: Contributions to the Relationship Between Personality and Psychopathology. European Journal of Psychological Assessment, 17(3), 241-250. doi: http://dx.doi.org/10.1027//1015-5759.17.3.241

Sandín, B., Valiente, R. M., Chorot, P., Santed M. A. y Lostao, L. (2008). SA-45: forma abreviada del SCL90. Psicothema, 20(2), 290-296.

Schmitz, N., Hartkamp, N., Kiuse, J., Franke, G. H., Reister, G. y Tress, W. (2000). The Symptom 
Check-List-90-R (SCL-90-R): A German validation study. Quality of Life Research: An International Journal of Quality of Life Aspects of Treatment, Care \& Rehabilitation, 9(2), 185-193. doi: http://dx.doi.org/10.1023/A:1008931926181

Stover, J. B., Castro Solano, A. y Fernández Liporace, M. (en prensa). Personality Assessment Inventory: Psychometric Analyses of the Argentinean Version. Psychological Reports.

Torres, L., Miller, M. J. y Moore, K. M. (2013). Factorial Invariance of the Brief Symptom Inventory18 (BSI-18) for Adults of Mexican Descent Across Nativity Status, Language Format, and Gender. Psychological Assessment, 25(1), 300-305. doi: http://dx.doi.org/10.1037/a0030436

Urbán, R., Kun, B., Farkas, J., Paksi, B., Kökönyei, G., Unoka, Z. y Demetrovics, Z. (2014). Bifactor structural model of symptom checklists: SCL-90-R and Brief Symptom Inventory (BSI) in a non-clinical community sample. Psychiatry Research, 216(1), 146-154. doi:

http://dx.doi.org/10.1016/j.psychres.2014.01.027

Vassend, O. y Skrondal, A. (1999). The problem of structural indeterminacy in multidimensional symptom report instruments. The case of SCL-90-R. Behaviour Research and Therapy, 37(7), 685-701. doi: http://dx.doi.org/10.1016/S0005-7967(98)00182$\mathrm{X}$

Zack, M., Toneatto, T. y Streiner, D. L. (1998). The SCL-90 factor structure in comorbid substance abusers. Journal of Substance Abuse, 10(1), 1998, 85-101. doi: http://dx.doi.org/10.1016/S08993289(99)80143-6

Zhang, J. y Zhang, X. (2013). Chinese college students' SCL-90 scores and their relations to the college performance. Asian Journal of Psychiatry, 6(2), 134140. doi: http://dx.doi.org/10.1016/j.ajp.2012.09.009 
